Themenheft Nr. 44: Datengetriebene Schule.

Forschungsperspektiven im Anschluss an den 27. Kongress der DGfE

Herausgegeben von Mandy Schiefner-Rohs, Sandra Hofhues und Andreas Breiter

\title{
Adaptive Lernsysteme zwischen Optimierung und Kritik
}

\section{Eine Analyse der Medienkonstellationen bettermarks aus informatischer und medienwissenschaftlicher Perspektive}

\author{
Andreas Weich $^{1}$ (D), Philipp Deny ${ }^{1}$ (D), Marvin Priedigkeit ${ }^{1}$ (D), Jasmin Troeger $^{1}$ (D) \\ ${ }^{1}$ Georg-Eckert-Institut - Leibniz-Institut für internationale Schulbuchforschung
}

\begin{abstract}
Zusammenfassung
Die Entwicklung und der Einsatz datengetriebener adaptiver Lernsysteme finden in einem komplexen Spannungsfeld statt. Informatische Herangehensweisen treffen auf schulische Wirklichkeiten, die sie zu spezifischen Bedingungen modellieren. Dabei sind Entwicklung und Einsatz adaptiver Lernsysteme sowohl mit Optimierungserwartungen als auch mit Kritik seitens der schulischen Akteure verbunden. Der Artikel analysiert die adaptive Mathematik-Lernplattform bettermarks vor diesem Hintergrund als Medienkonstellation, in die sich informatische Modellierungs-, Prozessierungs- und Optimierungsprinzipien einschreiben und mit schulischen Unterrichtspraktiken und Subjektpositionen verflechten. Zentrale Aspekte sind dabei, dass sich in bettermarks Logiken des Computational Thinking, des Solutionismus, der Programmierung iterativer bedingter Schleifen und der profilbasierten Repräsentation von Schülerinnen und Schülern, strikte Lernzielorientierung und hierarchische Machtverhältnisse auffinden lassen. Dabei entsteht eine Medienkonstellation, die weniger auf eine Adaption des Lernsystems an sich als auf die behavioristisch anmutende Adaption des lernenden Subjekts im Sinne der optimierenden Anpassung an vorgegebene Leistungsprofile abzielt. Gleichzeitig wird jenseits der konkreten Modellierungen in bettermarks die informatische Modellierung selbst kritisch in den Blick genommen, insofern sie systematisch nicht-komputierbare Elemente ausschliesst. Für eine Gestaltung adaptiver Lernsysteme, die diese Ergebnisse kritisch-reflexiv einbezieht, wird abschliessend vorgeschlagen, medienwissenschaftliche und informatische Akteure in partizipativen Projekten mit Lehrenden und Lernenden zusammen zu bringen.
\end{abstract}




\title{
Adaptive Learning Systems Between Optimization and Critique. An Interdisciplinary
} Media Constellation Analysis of Bettermarks

\begin{abstract}
The development and implementation of data-driven adaptive learning systems take place within a complex constellation. Principles from computer science come together with school realities that they transfer into specific formalized and computational models. In this context, the development and use of adaptive learning systems are associated with both optimization expectations and criticism on the part of school stakeholders. Against this backdrop, the paper analyzes the adaptive mathematics learning platform bettermarks as a media constellation that is pervaded by principles of modeling, processing and optimization that are typical for computer science and technology, and that are at the same time entangled with school practices of teaching and learning and established subject positions. Findings are that bettermarks is based on principles of computational thinking, solutionism, iterative conditional loops and student profiles that focus on learning objectives and hierarchical power relations. It constitutes a media constellation that is not primarily establishing an adaption of the system itself but a behavioristic adaption and optimization of the student according to given performance profiles. At the same time, beyond the concrete modelling in bettermarks, the informatics modelling itself is critically examined insofar as it systematically excludes non-computable elements. For a design of adaptive learning systems that critically and reflectively incorporates these results, it is finally proposed to bring together media science and informatics actors in participatory projects with teachers and learners.
\end{abstract}

\section{Einleitung}

«Das machen wir noch viel zu wenig, denn das ist aus meiner Sicht die Zukunft, eigentlich. Wir waren ja schon beim Thema Individualisierung der Lernprozesse. Und wenn man da so ein Tool an die Hand bekommen würde, auch gerade im Bereich von Übungsaufgaben, was den Lernstand automatisch adaptiert und vielleicht auch mithilfe von künstlicher Intelligenz arbeitet, kann das glaube ich sehr, sehr hilfreich sein. Das kann ein Lehrer nicht leisten.» (Eine Lehrperson)

«Das ist ja immer so eine Art Testsituation für die Schüler. [...] Wenn die Schüler merken, sie werden getestet und das wird dann dokumentiert, dann verhalten sie sich anders, als würde man lernen. » (Eine andere Lehrperson) 
Ich würde gern adaptive Lernsysteme benutzen, denn jeder hat ja Problemaufgaben. Und wenn die Mehrheit ein Thema nicht versteht, aber man selbst versteht es, aber ein anderes Thema nicht, dann ist es schlecht, wenn der Unterricht immer auf die Mehrheit angepasst wird. Schlecht wäre aber auch, wenn andere Schüler über das System sehen, dass man nicht gut hinterher kommt. (Eine Stimme aus der Schülerschaft, sinngemäss kombiniert aus einem Gruppeninterview)

Datengetriebene ${ }^{1}$ adaptive Lernsysteme, das deuten die Statements aus unseren Interviews ${ }^{2}$ an, stehen in einem komplexen Spannungsfeld. Die Lehrenden und Lernenden artikulieren Erwartungen hinsichtlich der Individualisierung von Lernprozessen, der Verbesserung des Umgangs mit heterogenen Gruppen, der Inklusion und der Entlastung der Lehrenden. In Zeiten der Covid-19-Pandemie kommen weitere Erwartungen im Hinblick auf Selbst- und Distanzlernen hinzu. Gleichzeitig adressieren die Lehrenden und Lernenden mögliche Probleme, z. B. im Hinblick auf die Vertraulichkeit und Transparenz der erhobenen Daten und der Veränderung von Lernpraktiken durch Testsituationen und Kontrollpraktiken. Auf der einen Seite geht es um Optimierung von Lernen und Unterricht als Zielvorstellung, auf der anderen Seite um eine kritische Reflexion dessen. All dies ist zudem vor dem Hintergrund der Tatsache zu sehen, dass informatische Systeme spezifische Modellierungen schulischer Wirklichkeiten mit sich bringen. Beispielsweise wurden Konzepte der Performativität, der (Un-)Sichtbarkeiten und der «Datendoubles» von Anwendenden dieser datengetriebenen adaptiven Systeme bereits in internationalen Forschungsarbeiten um Datafizierungsprozesse in der Bildungspraxis insbesondere im angloamerikanischen Raum diskutiert. In Anlehnung an Ball und Olmedo (2013) argumentieren beispielsweise Bradbury und Roberts-Holmes (2017), dass Daten eine wichtige Rolle in Prozessen der Performativität im aktuellen Bildungssystem spielen, sowohl in Bezug auf die Praxis als auch bezogen auf die Identität von Lehrpersonen und Lernenden;

«[...] the ways in which lists, forms, grids and rankings work to change the meaning of educational practice - what it means to teach and learn - and our sense of who we are in terms of these practices - what it means to be an educator, and to be educated». (Ball und Olmedo 2013, 6)

1 Unter «datengetrieben» verstehen wir solche Systeme, die Daten als Grundlage für die Erstellung oder Anpassung von Inhalten nutzen. Es geht also nicht um eine technikdeterministische Behauptung, dass das Vorhandensein von Daten die Nutzung oder Entwicklung der Systeme «treibt».

2 Die Forschung findet im Rahmen der Nachwuchsforschungsgruppe «Postdigitale Medienkonstellationen in der Bildung» statt, die von der Leibniz-Gemeinschaft und dem Niedersächsischen Ministerium für Wissenschaft und Kultur gefördert wird. http://www.gei.de/abteilungen/mediale-transformationen/postdigitale-medienkonstellationen.html. 
Auch Williamson (2015) sowie Fenwick, Mangez und Ozga (2014) greifen das Argument der Verwobenheit von Daten und der Steuerung von Bildung auf. Wie Williamson erörtert, werden Kinder durch ihre «Datendoubles» oder «Datendoppelgänger» repräsentiert, und zwar durch einen Prozess, der Bildung «maschinenlesbar» macht. Leistungsdaten der Kinder sind für das Steuern und Evaluieren der Schule zentral geworden, so dass die Kinder selbst auf statistische Daten reduziert werden (Bradbury und Roberts-Holmes 2017, 11), die dann auf die Identitäten im Klassenzimmer «zurückfallen».

In ihrer Argumentation um die Rekonstruktion von Identitäten durch Maschinen weisen Bradbury und Roberts-Holmes (2017) auch auf die Reproduktion von Ungleichheiten hin, indem sie auf die Ein- und Festschreibungskräfte von Daten eingehen, welche bei der Aufrechterhaltung der Differenzkategorien, welche den schulischen Diskurs durchdringen, beitragen:

«[...] terms used in data analysis such as 'summer- born', 'SEN' and 'EAL' define groups of children as problematic, more likely to experience 'underachievement'. The classification of children into these groups in databases makes the categories real, permanent and productive of certain subjectivities.»

Die informatische Modellierung schulischer Praktiken, Subjekte und Inhalte und die dabei sich vollziehenden Einschreibungen spezifischer Wissensbestände analytisch und differenziert zu skizzieren scheint hilfreich und notwendig, um sowohl die Entwicklung und Implementierung als auch den praktischen Einsatz adaptiver Lernsysteme reflektiert zu gestalten. Ziel des Beitrags ist es, hierfür einen Vorschlag zu unterbreiten und an einem exemplarischen Gegenstand - der Lernplattform bettermarks - zu erproben. Ausgehend von einer begrifflichen Bestimmung adaptiver Lernsysteme und der daran anknüpfenden Vorstellung des Gegenstands bettermarks werden wir eine medienwissenschaftliche Analyseperspektive auf adaptive Lernsoftware konturieren und im Anschluss grundlegende informatische Herangehensweisen problemlösender Modellierung skizzieren. Darauf aufbauend analysieren wir ausgewählte Funktionen von bettermarks und arbeiten abschliessend kritische Perspektiven auf den Gegenstand heraus sowie denkbare Alternativen. 


\section{Bettermarks als exemplarisches datengetriebenes adaptives Lernsystem}

Auch wenn datengetriebene adaptive Lernsysteme - insbesondere im schulischen Kontext - noch immer eine geringe Verbreitung haben ${ }^{3}$, ist das Angebot relativ divers. Sie alle eint zunächst, wenn man der Definition von Alexandros Paramythis und Susanne Loidl-Reisinger (2004, 182) folgt, eine Reihe spezifischer Eigenschaften:

«A learning environment is considered adaptive if it is capable of: monitoring the activities of its users; interpreting these on the basis of domain-specific models; inferring user requirements and preferences out of the interpreted activities, appropriately representing these in associated models; and, finally, acting upon the available knowledge on its users and the subject matter at hand, to dynamically facilitate the learning process.»

In Anlehnung an eine heuristische Liste von Merkmalen für Learning Analytics Systeme, die wir an anderer Stelle aufgestellt haben (Priedigkeit, Weich, und Schiering 2021), unterscheiden sich die Systeme sowie teilweise auch einzelne Funktionen von Systemen jedoch hinsichtlich des Zieles, das mit Adaption verfolgt wird (z. B. frühzeitig Hinweise auf einen möglichen Dropout zu geben, um ihn vermeiden zu können), hinsichtlich der Zielgruppe (z. B. Schülerinnen und Schüler, Studierende, mittelbar aber auch Lehrende, Eltern, Entscheidungsträgerinnen und Entscheidungsträger), hinsichtlich von Datenquellen (z. B. Antworten auf Aufgabenstellungen, Nutzungsfrequenzen, Verweildauern), der Art des in der Adaption enthaltenen Feedbacks (z. B. Bereitstellen von Aufgaben, Geben von Hinweisen), sowie hinsichtlich der Art und Weise, wie die Daten zum Hervorbringen der Adaption prozessiert werden (z. B. regelbasiert oder mittels Machine Learning), und auch darin, welchen Aspekten von Security und Privacy Rechnung getragen wird (z .B. ob überhaupt oder auch differenziert wird im Sinne der Privacy Protection Goals: Vertraulichkeit, Transparenz, Integrität, Verfügbarkeit, Interventionsmöglichkeit, Nicht-Verknüpfbarkeit; vgl. Hansen, Jensen und Rost 2015).

Eine andere Systematisierung liefert Lehmann $(2010,19)$ und unterscheidet «(1) die durchführende Instanz, (2) die Quelle des Entscheidungsinputs, (3) die Taktung der Adaption, (4) der Gegenstand der Adaption, (5) der Zweck der Adaption.»

Je nach Zusammenstellung der Ausprägungen dieser Merkmale ergeben sich unterschiedliche Bezüge zum oben skizzierten Spannungsfeld. Im Rahmen dieses Artikels können selbstverständlich nicht alle in den Blick genommen werden. Stattdessen werden wir mit bettermarks ein System exemplarisch analysieren, das sich nicht nur hinsichtlich der eben genannten Merkmale auszeichnet, sondern auch durch eine national und auch international relativ hohe Verbreitung (knapp 800 Schulen in

3 ICIL 2018 (vgl. Eickelmann et al. 2019): Die Lehrpersonen nutzen häufiger digitale Medien im Unterricht als vor fünf Jahren, und digitale Kompetenzen spielen in den Lehrplänen eine grössere Rolle. 60 Prozent nutzen digitale Medien mindestens einmal pro Woche. Dieser Anteil hat sich im Vergleich zu 2013 fast verdoppelt. 23,2 \% nutzen digitale Medien sogar täglich. 2013 waren es nur 9,1 \%. 
DE, AT \& CH setzen bettermarks ein, Hamburg als erstes Bundesland in der Breite). Des Weiteren ist bettermarks in der Durchführung des renommierten Mathematikwettbewerbs Känguru involviert. Betrachtet man die in deutschen Unterrichtspraxen verwendeten Systeme (Klassifizierung in Anlehnung an Bulger 2016, Groff 2017 und Troeger in Vorb.), nimmt bettermarks aber nicht nur hinsichtlich der hohen Verbreitung eine Sonderstellung ein, sondern ist auch in Bezug auf das Adaptivitätslevel als exemplarisches Beispiel prädestiniert. ${ }^{4}$ Die bettermarks $\mathrm{GmbH}$ wurde laut eigenen Angaben (bettermarks 2020a) 2008 gegründet, ihre Plattform ist in mehrere Sprachen übersetzt und in zehn Ländern bei über 100.000 Schülerinnen und Schüler pro Monat im Einsatz. Die Plattform ermöglicht es Lehrenden, ihren Schülerinnen und Schülern im Klassenverband oder individuell Aufgaben zu geben. Nach der Bearbeitung einer jeden Aufgabe bekommen die Lernenden Feedback über die Richtigkeit ihrer Antworten, ggf. Hilfestellungen und Erklärungen, sowie weitere Übungsaufgaben, sofern eine sogenannte «Wissenslücke» diagnostiziert wurde. Die Lehrenden können sich über die Bearbeitung der Antworten und «Wissenslücken» der einzelnen Schülerinnen und Schüler informieren sowie statistische Auswertungen der gesamten Klasse durchführen. Zugleich bekommen sie auch Vorschläge für konkrete Aufgaben, um die «Wissenslücken» der Schülerinnen und Schülern zu «schliessen».

Im Hinblick auf die Charakteristika von Adaptivität werden die Antworten der Schülerinnen und Schüler auf die gestellten Aufgaben als Quelle des Entscheidungsinputs genutzt. Die Art und Weise der Prozessierung der Nutzungsdaten ist anhand der Analysen der Programmoberflächen heraus nicht eindeutig zu rekonstruieren, doch es liegt nahe, dass in erster Linie ein User Modeling mit regelbasierten Adaptionsverfahren kombiniert wird. Das Ziel der Adaption ist das Erreichen gegebener Lernziele. Gegenstand der Adaption bzw. Art des Feedbacks ist die Diagnose von «Wissenslücken» sowie die Unterbreitung darauf bezogener Lernangebote in Form von Aufgaben und Hilfestellungen. Die Adressatinnen und Adressaten der aus den Nutzungsdaten generierten Ergebnisse sind zum einen die Lernenden, insofern sie adaptive Lernangebote bekommen, aber auch die Lehrenden, insofern sie über den Lernstand der Schülerinnen und Schüler informiert werden und ihrerseits über das System daraufhin diesen spezifische Lernangebote unterbreiten können. Die durchführende Instanz der Adaption ist demnach sowohl das System, gesetzt dem Fall, dass es adaptiv Inhalte generiert, als auch die Lehrperson, sollte sie das System als adaptierbares nutzen, um ihrerseits Lernangebote für Schülerinnen und Schüler zusammenzustellen.

4 Eine detaillierte Übersicht der in Deutschland verwendeten Systeme befindet sich in Vorbereitung (Troeger, i. Vorb.). 


\subsection{Medienkonstellationsanalytischer Verfahrensvorschlag}

Um die bettermarks-Plattform analytisch in den Blick zu nehmen, soll sie im Folgenden als Medienkonstellation modelliert werden. Das Medienkonstellationsmodell geht davon aus,

«dass Medien sich nicht als Objekte mit bestimmbaren Eigenschaften definieren lassen, sondern Medialität sich nur in heterogenen Konstellationen konstituiert. [...] Das Modell geht weiterhin davon aus, dass diese Konstellationen sich heuristisch durch ein Zusammenspiel von Elementgruppen beschreiben lassen: Materialitäten, Wissen/Praktiken, Inhalte und Subjektpositionen.» (Weich, Koch, und Othmer 2020, 44f.)

Es ist auf theoretischer Ebene an eine Vielzahl von Ansätzen anschlussfähig, die gegenwärtig in der Medienpädagogik eine Rolle spielen: von medientheoretischen über poststrukturalistische diskurs-, dispositiv- und praxistheoretischen, akteurnetzwerk-theoretischen bis zu jenen der Postdigitalität (vgl. dazu ausführlicher Weich, Koch, und Othmer 2020; sowie Weich 2020).

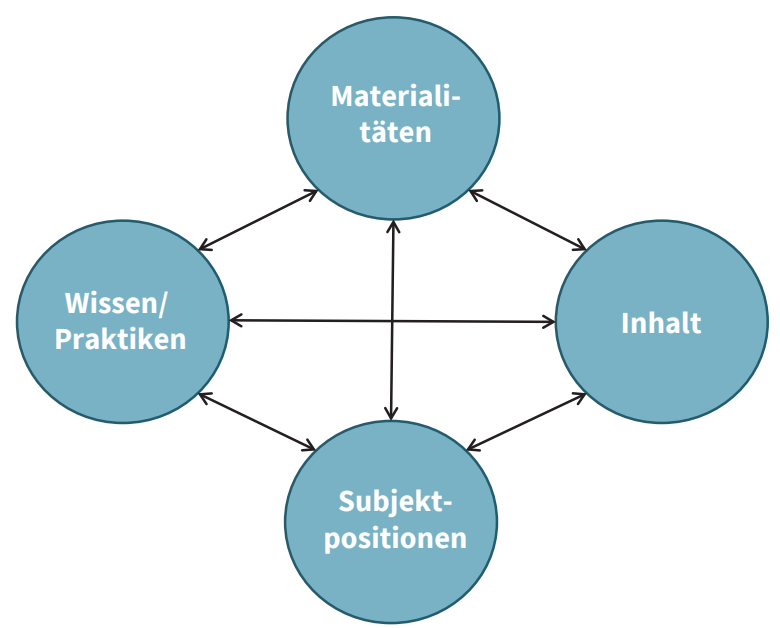

Abb. 1.: Medienkonstellationsmodell (eigene Darstellung).

\section{Das Modell ist dabei}

«als Heuristik zu verstehen, die einerseits die Modellierung von Medien ohne eine verkürzende Identifikation mit der (insbesondere digitalen) Technik, und andererseits die Analyse der derart modellierten Medien anhand eines einfachen Rasters erlaubt» (Weich 2020; Herv. im Original).

Im Folgenden wird es also darum gehen, bettermarks als Zusammenspiel zwischen Materialitäten (z. B. Computertechnologie), Wissen/Praktiken (z. B. Gestaltungsprinzipien, informatische Modelle, didaktische Konzepte), Inhalten (z. B. 
fachliche Inhalte, aber auch die Strukturen, Oberflächen und Interfaces des Frontends der Software) und Subjektpositionen (d. h. in diesem Fall die Funktionsstellen, die für Lehrende und Lernende entworfen werden) zu bestimmen. Im Hinblick auf adaptive Lernsysteme kann hierbei angeschlossen werden an bestehende Überlegungen zu soziomateriellen Praktiken, Subjektivierungsweisen, aber auch Gestaltungsprinzipien im Kontext von Learning Analytics (exempl. Allert, Asmussen, und Richter 2018, Allert und Richter 2017, Weich 2018). Dabei legen wir folgende Leitfragen zugrunde: Welches Wissen und welche Praktiken (insb. informatische und pädagogisch/didaktische), die in die Software eingeschrieben sind, lassen sich an der Oberfläche (Inhalte) rekonstruieren und wie werden sie modelliert? Wie werden sie und Subjekte durch die Software (Inhalte) modelliert und welche Subjektpositionen werden adressiert? Welche Rolle spielt Computertechnologie (d. h.z. B. Prozessoren, Speicher und Netzwerke) als materielle Möglichkeitsbedingung für die Inhalte und als diskursiv aufgeladene Instanz?

Der Analysefokus liegt also auf der Ebene der Inhalte (in diesem Fall dem Frontend der Software sowie Informationen über die Software aus den Selbstdarstellungen der Firma), die dahingehend betrachtet werden, welche Einschreibungen sich daraus ablesen lassen und wie die Inhalte sowie in geringerem Masse auch die Materialitäten (hier abstrakt verstanden als Computertechnologie) vor diesem Hintergrund Subjekte adressieren und positionieren sowie Praktiken modellieren.

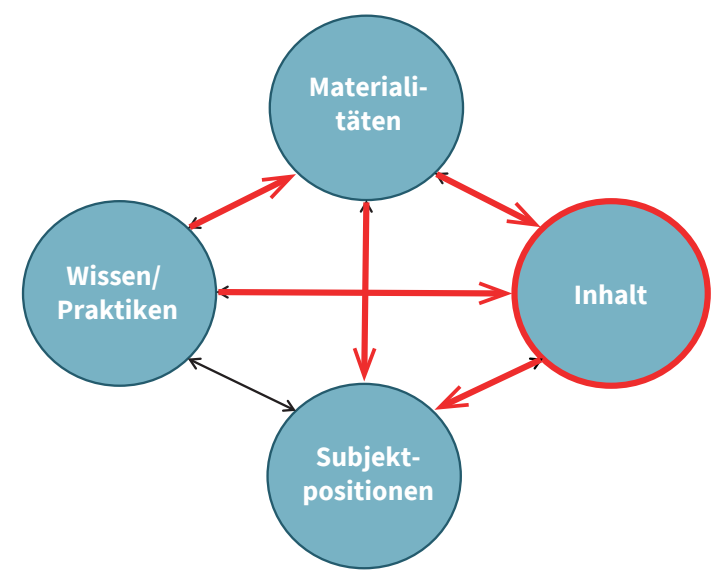

Abb. 2.: Fokussierte Wechselwirkungen für die Analyse von bettermarks (eigene Darstellung).

Dieser medienkulturwissenschaftliche Ansatz vermeidet naive Betrachtungsweisen von Medien, die (Lehr-/Lern-)Inhalte losgelöst von ihren medialen Existenzbedingungen analysieren und bindet explizit die Frage nach technischen Voraussetzungen und Konsequenzen sowie subjekt-, diskurs- und kulturbezogene Fragen ein. Gleichzeitig vermeidet er durch die Berücksichtigung von Wissen und Praktiken technikdeterministische Annahmen, die in den Begriff des «Datengetriebenen» durchaus 
hineingelesen werden könnten. Lerntheoretische Anschlüsse ergeben sich dadurch, dass sich die Modellierung der jeweiligen Praktiken und Subjektpositionierungen mit entsprechenden Diskursen zu Behaviorismus, Kognitivismus und Konstruktivismus (vgl. überblicksartig Reinmann 2013) in Bezug setzen lässt. Bildungs- und medienbildungstheoretische Anschlüsse ergeben sich dadurch, dass mit Medienkonstellationen die medialen Voraussetzungen für Artikulationen und Konstruktionen von Selbst- und Weltverhältnissen (vgl. Jörissen und Marotzki 2009) analytisch beschrieben werden können. Aus Gründen der Fokussierung und geschuldet dem aktuellen (Zwischen-)Stand unserer Forschung bleiben in diesem Artikel einige dieser Perspektiven (noch) aussen vor, die über eine Medienkonstellationsanalyse ebenfalls adressierbar sind, wie z. B. Nutzungs- und Aneignungspraktiken situierter Subjekte oder tiefergehende Analysen der zugrunde liegenden Medientechnik, die über Feldstudien, Interviews mit Schülerinnen und Schüler, Lehrpersonen und Entwicklerinnen und Entwickler sowie Einblicke in Software angestellt werden. Gleichzeitig wären in zusätzlichen Analysen weitere adaptive Systeme als Medienkonstellationen zu analysieren und miteinander ins Verhältnis zu setzen. Dies stellen aus unserer Sicht Desiderate für eine umfassendere Analyse dar, für die das vorliegende Paper eine erste Grundlage liefern kann.

\subsection{Problemlösende Modellierung als grundlegende informatische Herangehensweise}

Die konkrete informatische Implementierung eines datengetriebenen adaptiven Lernsystems aus dem fertigen Produkt abzulesen, ist nahezu unmöglich. Es gibt jedoch grundlegende Prinzipien, die in der Informatik und der Softwareentwicklung im Regelfall angewendet werden (vgl. exemplarisch Modrow und Strecker 2016, 88ff.). Ein zentrales Prinzip ist dabei die Modellierung realweltlicher Phänomene als über Komputation zu lösende Probleme (Poole und Mackworth 2017, 12).

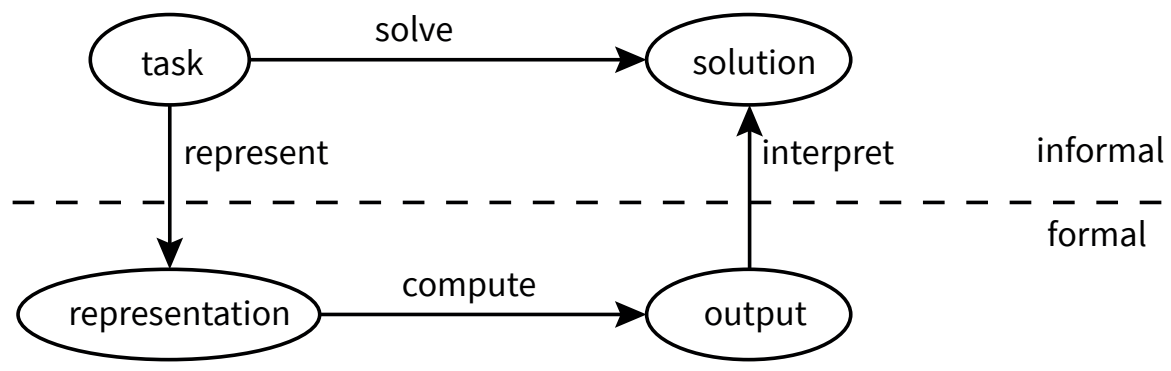

Abb. 3.: Informatischer Modellierungsprozess (vgl. Poole und Mackworth 2017).

Am Beginn steht die nicht-formale Beschreibung einer Aufgabe (task) sowie einer dafür vorgesehenen Lösung (solution). Um die Lösung mit informatischen Mitteln erreichen zu können, muss zunächst die Aufgabe (task) formal repräsentiert werden 
(represent). Die daraus hervorgehende formale Repräsentation der Aufgabe (representation) zeichnet sich durch ihre Komputierbarkeit aus (compute), durch die ein formales Ergebnis (output) erzeugt werden kann. Dieses dient als Grundlage für eine Art Rückübersetzung in den nicht-formalen Bereich (interpret), die das ursprüngliche Problem zu lösen hilft. Der Aspekt der Optimierung liegt dabei darin, dieses Problem bestmöglich mittels der Repräsentation und deren Komputierung zu lösen.

Dieses sehr generische Prinzip liegt auch dem Ansatz des Computational Thinking zugrunde, das - vereinfacht gesagt - eine aus der Informatik abgeleitete Art und Weise ist, sich die Welt zu erschliessen. Jeanette Wing, eine aktuell prominente Vertreterin dieses Ansatzes, schreibt in diesem Sinne:

«Computational thinking involves solving problems, designing systems, and understanding human behavior, by drawing on the concepts fundamental to computer science. [...] Having to solve a particular problem, we might ask: How difficult is it to solve? and What's the best way to solve it? [...] Computational thinking is using abstraction and decomposition when attacking a large complex task or designing a large complex system. It is separation of concerns. It is choosing an appropriate representation for a problem or modeling the relevant aspects of a problem to make it tractable.» (Wing 2006, 33)

Dabei betont sie die Nähe der informatisch geprägten Welterschliessung zu Mathematik und Ingenieurwesen:

"Computer science inherently draws on mathematical thinking, given that, like all sciences, its formal foundations rest on mathematics. Computer science inherently draws on engineering thinking, given that we build systems that interact with the real world. The constraints of the underlying computing device force computer scientists to think computationally, not just mathematically.» (ebd., 35)

Bezogen auf das Medienkonstellationsmodell lassen sich zusammenfassend als grundlegende und typische Wissensbestände und Praktiken Problemlösung, Optimierung, Modellierung, Repräsentation, Komputation, Mathematik, «engineering» sowie eine Bindung an die «underlying computing device» (Wing 2006, 35), also die Hardware als ermöglichende und einschränkende Materialität, ableiten. In der konkreten Umsetzung kommen je nach System verschiedene typische Werkzeuge wie bedingte Anweisungen (z. B. durch IF/THEN/ELSE-Strukturen), iterative Schleifen, Graphen, Datenstrukturen u.a.m. zum Einsatz, die jedoch nicht an dieser Stelle, sondern bei ihrem jeweiligen Auffinden bei der Analyse von bettermarks erläutert werden. 


\section{Analyse ausgewählter Funktionen und Strukturen von bettermarks}

Im Folgenden werden ausgewählte Funktionen von bettermarks vor dem Hintergrund des Medienkonstellationsmodells und der skizzierten informatischen Wissensbestände und Praktiken beschrieben und analysiert.

\subsection{Lernzielnetz}

Eine Grundlage der Strukturierung des Lernangebots in bettermarks ist das sogenannte «Lernzielnetz» (s. Abb. 4 und 5). Es definiert Lernziele, ordnet sie in mathematische Themenfelder ein und verknüpft sie in Form von Abhängigkeiten miteinander. Letztere gehen davon aus, dass jedes Lernziel das Erreichen anderer Lernziele zur Vorbedingung hat, wie Christophe Speroni, einer der Gründer von bettermarks, in einem Produktvideo ${ }^{5}$ erläutert: «Diese Lernziele sind vernetzt. Das bedeutet, wir wissen für jedes einzelne Lernziel, was das entsprechende Vorwissen ist. Also, bevor ich ungleichnamige Brüche addieren kann, sollte ich in der Lage sein, gleichnamige Brüche zu addieren, usw.» (bettermarks 2018a, 1:07-1:26).

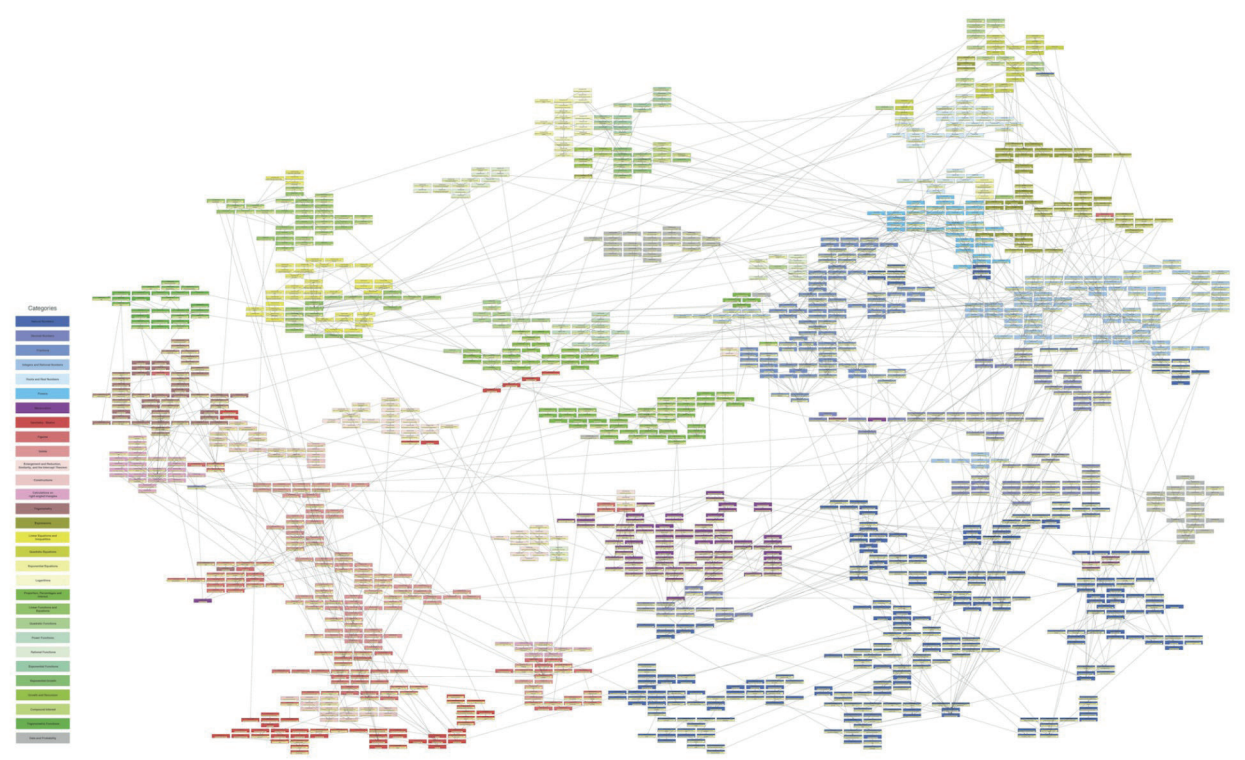

Abb. 4.: Diagramm des Lernzielnetzes von bettermarks (Quelle: https://de.bettermarks.com/ wp-content/uploads/2020/06/lernzielnetz.jpg).

5 Produktvideos als Quelle sagen selbstverständlich nur bedingt verlässlich etwas über die tatsächlichen Funktionsweisen aus, sind jedoch zum einen ein valider Anhaltspunkt dafür und zum anderen Teil der Adressierung der Lehrenden und Lernenden sowie ein Beleg der in die Medienkonstellation eingehenden Wissensbestände. Mit Hartmut Winkler (1997) könnten einige der Aussagen in den Videos als «Wunschkonstellationen» veranschlagt werden, die im Sinne des zu Beginn des Textes skizzierten Spannungsverhältnisses bestimmte Erwartungen und Wünsche bzw. das Versprechen ihrer Erfüllung artikulieren. 


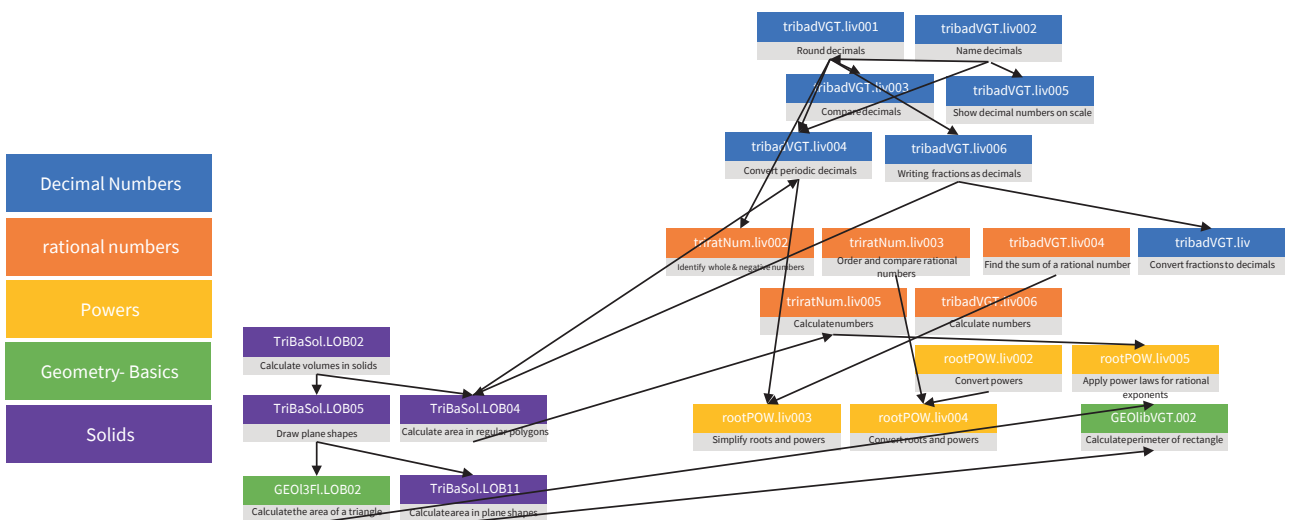

Abb. 5.: Vergrösserter Ausschnitt des Lernziel-Diagramms (Nachbildung von bettermarks 2018a, $0: 53)$.

Im oben skizzierten Prozess der informatischen Modellierung wählt bettermarks zu dem dazugehörigen «task» des Erlernens der Unterrichtsinhalte das Erreichen netzförmiger aufeinander aufbauender Lernziele als «representation» in Form eines Graphen. In diesem Modellierungsprozess wird mit «Vernetzung» eine Repräsentationsstruktur in die Inhalte eingeschrieben, die recht typisch für informatische Modellierungen ist; u. a. veranschlagen Döbeli-Honegger $(2017,16 \mathrm{ff}$.) Vernetzung und Modrow und Strecker $(2016,19 \mathrm{ff}$.) Vernetzbarkeit als Grundprinzipien von Computertechnologie und Informatik.

An dieser Stelle und auch im Folgenden geht es nicht um die Frage, ob die beschriebenen Modellierungen adäquat sind oder nicht, sondern um die Explizierung, dass es sich hierbei um eine durch spezifische (oftmals eng mit der Informatik verbundene) diskursive Wissensbestände geprägte, kontingente Setzung handelt. ${ }^{6}$

Jenseits dieser Struktur an sich schreiben sich auch innerhalb des Lernzielnetzes Wissensbestände ein. Basierend auf dem Domänenwissen, zum Beispiel den fachdidaktischen Diskursen, wird die konkrete Auswahl getroffen, welche Lernziele wie in den Graphen eingebettet werden.

Noch eine Ebene feiner granuliert als die Lernziele und auf die Dispositionen der Schülerinnen und Schüler bezogen sind die Kompetenzen, die ebenfalls miteinander vernetzt sind:

«Darüber hinaus haben wir auch Aufgabenschritte bzw. Kompetenzen, die ich innerhalb von Aufgabenschritten anwenden muss, ebenfalls vernetzt. [...] Anhand des Lernzielnetzes wissen wir nicht nur, was das unmittelbare Vorwissen eines Lernzieles ist. Wir wissen auch, was für eine Übung wir zusteuern müssen, wenn es bei einem bestimmten Rechenschritt hapert». (bettermarks 2018a, 1:26-2:20)

6 Auf das Netz als Metapher und Wunschkonstellation medientheoretisch und -historisch einzugehen, würde an dieser Stelle den Rahmen sprengen, aber es sei verwiesen auf Winkler $(1997,14 \mathrm{ff}$.$) , Schröter (2004)$ und Gießmann (2014). 
Das Schüler-/Schülerinnen-Subjekt wird folglich als Profil aus mathematischen Kompetenzen repräsentiert, die entweder vorhanden sein können oder nicht. Auch hier finden wieder Setzungen auf mehreren Ebenen statt: Zum einen die Repräsentation des Subjekts in Form einer Zusammenstellung distinkter Merkmale, d. h. eines Profils. Auch wenn wir diese Repräsentationsform in vielen Kontexten antreffen und für selbstverständlich erachten, ist auch sie eine kontingente und speist sich aus einer Vielzahl von Diskursen und genealogischen Linien (vgl. Weich 2017). In der Informatik wird für derartige Modellierungen häufig das entity-relationship model (Chen 1976) herangezogen. Die Schülerin/der Schüler wird in diskreten Einheiten repräsentiert, also im Wortsinne digitalisiert, was ihn/sie überhaupt erst an das informatische System anschlussfähig, weil komputierbar macht. Zum anderen ist die Wahl dessen, was als Merkmal ein- und ausgeschlossen wird, eine Setzung. Die Modellierung des Subjekts als Zusammenstellung von Kompetenzen ist kontingent und lässt sich u. a. mit Diskursen der Kompetenzorientierung in Verbindung bringen. Kompetenzen zeichnen sich dadurch aus, dass sie erworben oder zumindest gefördert werden können. Diese Vorstellung ermöglicht - im Gegensatz z. B. zu unveränderlichen Charaktermerkmalen oder genetischen Eigenschaften - die Forderung nach Arbeit am eigenen Kompetenzprofil. Im Rahmen von bettermarks ergibt sie sich durch die Verbindung der Kompetenzen mit dem «Lernzielnetz»: Wenn ich bestimmte Lernziele nicht erreicht habe, muss es an meinem defizitären Kompetenzprofil liegen, d. h. meine Kompetenzen (verstanden als meine realweltlichen Dispositionen) matchen nicht mit den in der formalen Repräsentation geforderten Kompetenzen (verstanden als Soll-Werte meines Nutzerprofils). Und da sich die Werte im Profil direkt aus den Eingaben des Subjekts in das System ergeben, wird es als verantwortlich für ihre zielgerichtete Anpassung adressiert. Die Subjektposition der Schülerin/des Schülers ist also geprägt durch das «Geprüft-Werden` als Testobjekt und den Imperativ zur Ausübung der Praktik einer Arbeit an sich selbst mit dem Ziel der Optimierung des eigenen Profils. Bettermarks schliesst damit zunächst an bestehende schulische Praktiken an, insofern Curricula Lernziele und Kompetenzen festlegen, die Schülerinnen und Schüler zu einem gewissen Zeitpunkt zu erreichen haben. Ein Nichterreichen dieser Ziele wird als Defizit, das es zu beheben gilt, verstanden. Dabei verabsolutiert das System jedoch die formale und komputierbare Repräsentation der Lernenden, sowie die nicht-verhandelbare Einpassung in das «Lernzielnetz» und erhöht in der Adressierung ihre Selbstverantwortlichkeit.

\subsection{Fehlerkultur, Wissenslücken und Wiederholungen}

Besonders deutlich wird diese Subjektpositionierung über die Konzepte des Lernens aus Fehlern, der «Wissenslücken» und der Wiederholung. Speroni (bettermarks 2018b) nennt als Vorbild für das Konzept von bettermarks ein «leeres Blatt Papier», 
auf dem man - anders als in Multiple Choice Fragen - «jeden erdenklichen Fehler machen» und daraus lernen könne (bettermarks 2018b, 1:55-2:05). Selbstverständlich sind die möglichen Inhalte der Lösungseingaben durchaus begrenzt und keinesfalls so frei wie auf einem leeren Blatt Papier (abgesehen davon, dass es einige Multiple Choice Fragen in bettermarks gibt und zudem auch auf einem leeren Blatt Papier nicht jeder erdenkliche Fehler gemacht werden kann). So sind je nach Aufgabe entweder Graphen, Formeln oder Zahlen möglich, Texte oder freie Zeichnungen jedoch nicht, da sie als Inhalte in der Modellierung nicht vorgesehen sind.

Die Freiheit, Fehler zu machen, ist zudem funktional eingebunden. Es dient nicht im Sinne einer Fehlerkultur der Förderung von Kreativität durch die Erlaubnis, auch riskante Versuche zu unternehmen, sondern der schrittweisen Annäherung an vorgegebene Ziele. Speroni schreibt dazu:

«Das pädagogische Konzept von bettermarks basiert darauf, es Schülerinnen und Schülern zu ermöglichen, aus ihren Fehlern zu lernen. Dies geschieht auf Basis folgender Interaktionen:

Aufgaben rechnen

Fehler machen

Feedback bekommen

Es erneut versuchen und das Gelernte anwenden.»

(bettermarks 2020a)

Dieser Ansatz ähnelt einer in der Informatik häufigen Form der Algorithmisierung durch bedingte Anweisungen und Schleifen. Das lernende Subjekt ist in eine Art iterierende IF/THEN/ELSE-Struktur eingebunden. Abstrakt formuliert: Wenn (If) die Lösung der Aufgabe richtig ist, dann (Then) gehe zur nächsten Aufgabe/zum nächsten Lernziel; wenn nicht (Else), versuch es erneut auf Grundlage des Feedbacks.

Eines der Produktvideos zeigt die technische Umsetzung dieser schrittweisen Annäherung, die ebenfalls nach der IF/THEN/ELSE-Logik prozessiert wird (wenn Multiplikationszeichen vorhanden, gib Rückmeldung X; wenn Variablen nicht zusammengefasst sind, gib Rückmeldung $Y$; wenn Variablen nicht alphabetisch sortiert sind, gib Rückmeldung Z). 


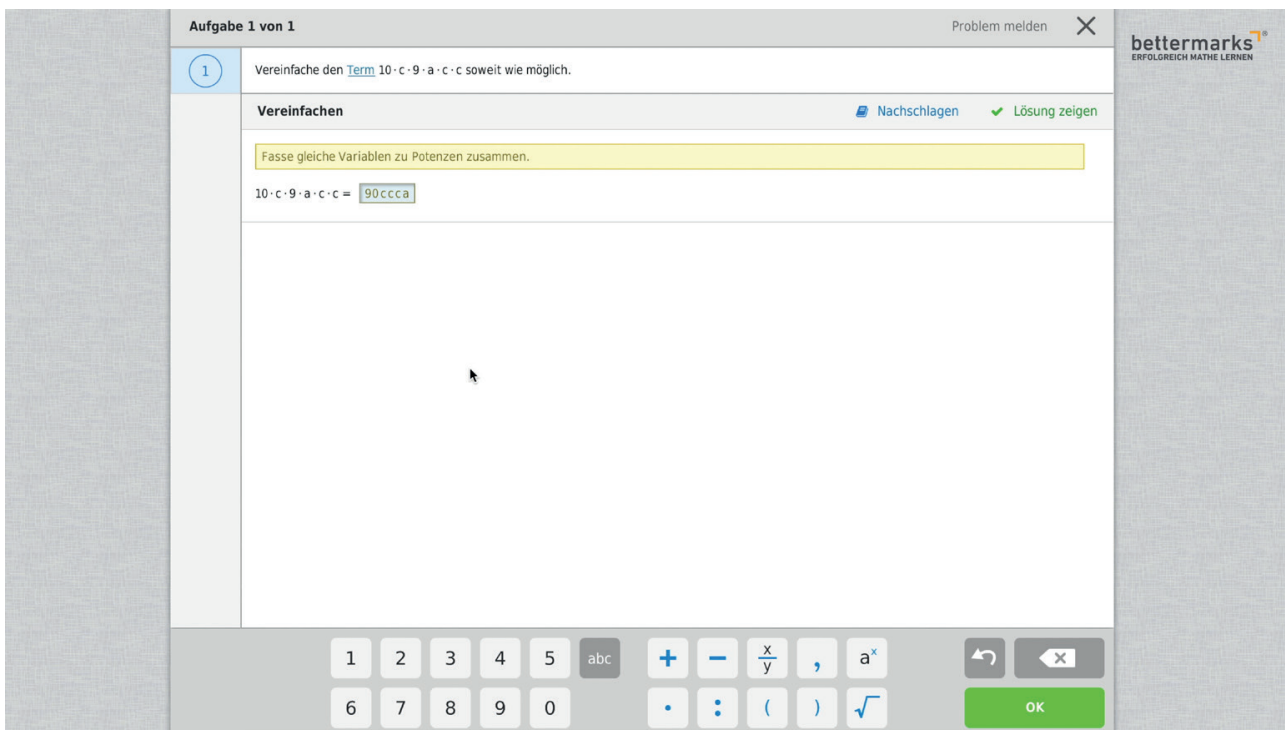

Abb. 6.: Rückmeldung auf fehlerhafte Eingabe in bettermarks (Quelle: bettermarks 2018b, 2:45).

Der prototypische Lösungsweg, der den Rückmeldungen (s. exempl. Abb. 6 und 7) zugrunde liegt, weist in derartigen Fällen selbst eine ähnliche Algorithmizität auf. Bei mathematischen Aufgaben und Lösungspraktiken gehen Form und Inhalt derartiger Programme oftmals sehr gut zusammen - nicht zuletzt, da Informatik und Mathematik systematisch und historisch bestimmte diskursive Praktiken miteinander teilen. Die Implementierung in bettermarks schreibt diese diskursiven Verschränkungen in seine Inhalte ein und formiert so einen informatisch/algorithmisch geprägten Zugang zu Mathematik. Bestimmte Formen der Algorithmisierung und des Computational Thinking werden so gewissermassen auch Vorbild für mathematische Lernprozesse. 


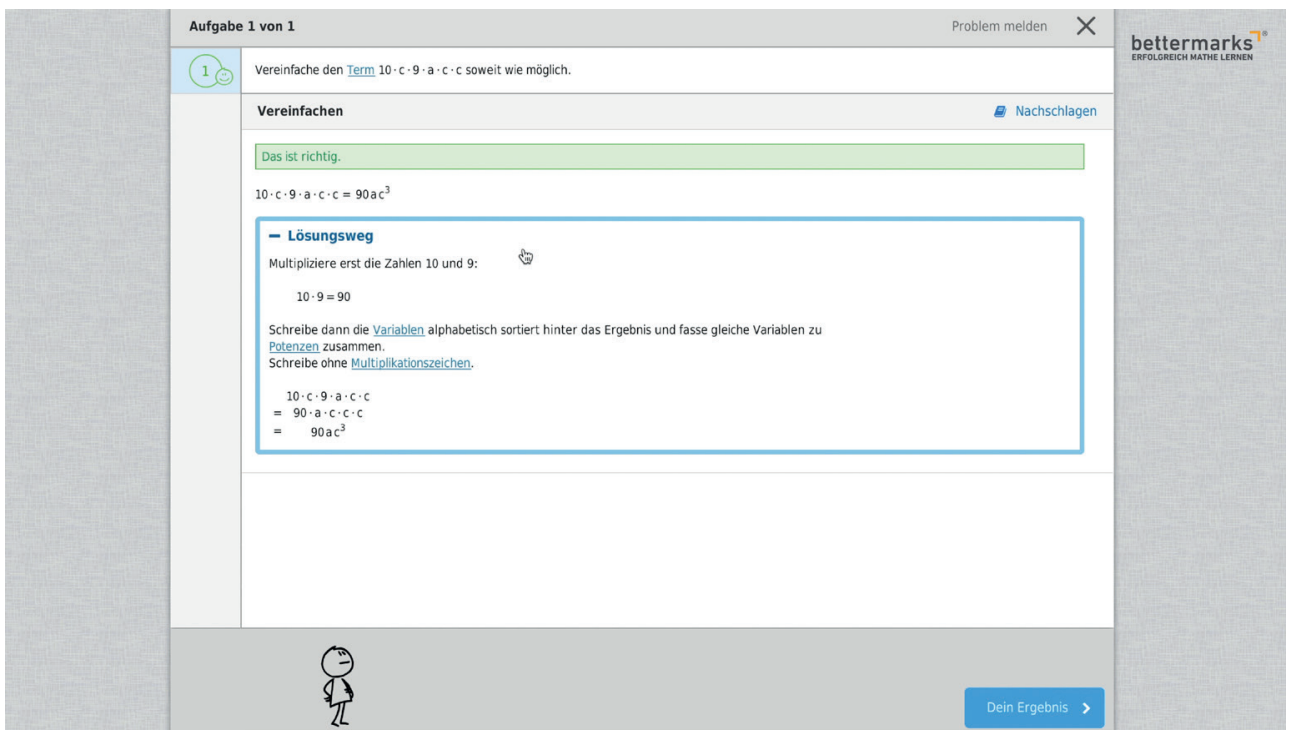

Abb. 7.: Anzeige eines Lösungswegs in bettermarks (Quelle: bettermarks 2018b, 3:17).

Ein zentrales Konzept bei bettermarks ist dabei jenes der «Wissenslücke». Sie repräsentiert eine defizitäre Disposition der Lernenden vor dem Hintergrund des «Lernzielnetzes»: Wenn sie Aufgaben, die ein bestimmtes Lernziel abbilden, wiederholt nicht richtig lösen können, wird im Modell angenommen, dass im «Lernzielnetz» eine Lücke zwischen den zuletzt erreichten Lernzielen und dem damit verknüpften, aber nicht erreichten Lernziel vorliegt. Die Schülerin/der Schüler kann die im «Lernzielnetz» hinterlegte Verbindung nicht nutzen, woraus das System eine Leerstelle innerhalb seines/ihres Kompetenznetzes ableitet. Dazu erklärt Speroni: «Angenommen, der macht hier immer wieder Fehler, dann registriert bettermarks das, erkennt eine Wissenslücke und stellt eine Übungsaufgabe zur Verfügung, um diese Wissenslücke zu schliessen.» (bettermarks 2018b, 9:20-9:29)

Die Formulierung, dass «Wissenslücken» «erkannt» werden, suggeriert dabei eine bestimmte Objektivität. Damit greift das Produktvideo auf diskursive Linien zurück, die Computer als objektive - weil formal, logisch und mathematisch präzise operierende - Diagnoseinstrumente veranschlagen. Die bisherigen Ausführungen sollten demgegenüber deutlich gemacht haben, dass die «Wissenslücken» ebenso wie ihr «Erkennen» keine diskursiv neutralen, objektiven Tatbestände, sondern Produkt der in das System eingeschriebenen Wissen/Praktiken und Subjektpositionierungen sind. Lernen als Praktik wird modelliert als Arbeit an vom System repräsentierten/ konstituierten Defiziten bis zum Erreichen des gewünschten Zustands. Der Fokus liegt auf der Frage, ob auf bestimmte Reize die gewünschten Reaktionen erfolgen. So ergibt sich eine Nähe zu jenen Anwendungen, die Krommer (2013) verschiedentlich als «Skinner Apps» bezeichnet hat, um ein wenig polemisch auf die in sie eingeschriebenen behavioristischen Wissensbestände und Praktiken hinzuweisen. Überspitzt 
formuliert ist in diesem Sinne nicht das Lernsystem die adaptive Komponente innerhalb der Medienkonstellation, sondern das lernende Subjekt, das sich als Variable innerhalb der IF/THEN/ELSE-Strukturen und des «Lernzielnetzes» an die Soll-Werte der Software adaptiert und in diesem Sinne optimiert (vgl. auch Weich 2018).

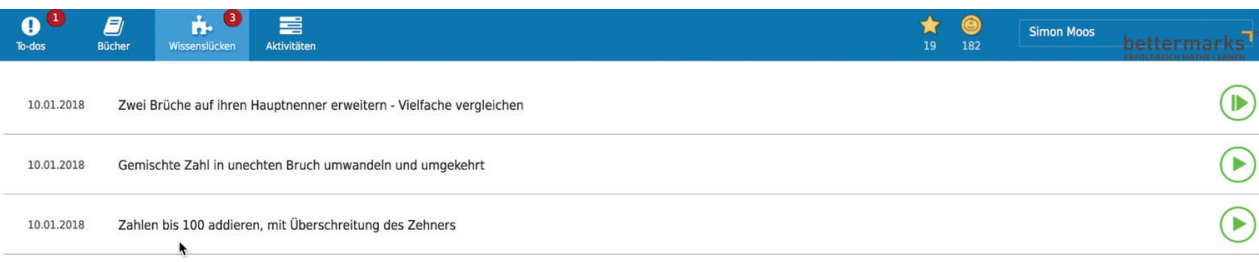

Abb. 8.: Anzeige der Wissenslücken eines Schülers oder einer Schülerin (Quelle: bettermarks 2018b, 9:39).

Die Verantwortung dieser optimierenden Adaption, verstanden als die Arbeit des «Schliessens» der konstatierten «Wissenslücken» (s. Abb. 8), wird in erster Linie den Schülerinnen und Schülern zugesprochen. Im ihrem Interface gibt es einen Bereich «Wissenslücken», in dem zur Erläuterung steht: «Hier siehst du deine Wissenslücken aus den Bereichen, die du besonders üben musst». Zudem wird in den Produktvideos die «Eigenständigkeit» der Schülerinnen und Schüler betont:

«Kann dann wirklich das üben, was ich nicht konnte. So, hab die Aufgabe richtig gelöst, bin jetzt fertig, hab meine Wissenslücke geschlossen und hab damit eigenständig den Anschluss gefunden. Und in Uruguay beispielsweise wird bettermarks ja im gesamten Land eingesetzt. Und dort werden $70 \%$ aller Wissenslücken innerhalb einer Woche erfolgreich geschlossen. Das bedeutet, dass 70\% der Schüler eigenständig es schaffen, den Anschluss zu finden. Und die 30\%, die es nicht schaffen, die kann man in der Auswertung identifizieren, und denen dann halt nochmal unter die Arme greifen, persönlich, und ihnen noch andere Hilfestellungen geben.» (bettermarks 2018b, 10:38-11:25)

Mit der «Eigenständigkeit» der Schülerinnen und Schüler geht neben der Subjektpositionierung durch die oben angesprochene Arbeit am eigenen Selbst zudem das Versprechen der Entlastung der Lehrperson einher, die sich um die «70\%» der mithilfe von bettermarks «geschlossenen Wissenslücken» nicht mehr kümmern müssen, und dadurch Kapazitäten gewinnen, um sich intensiver mit den verbleibenden 30\% auseinander zu setzen. Damit wird den Lehrenden eine veränderte Subjektposition zugewiesen: bettermarks, so zumindest das Versprechen, übernimmt bestimmte Fleissaufgaben im Bereich der individuellen Übungsanleitung, Überprüfung und Rückmeldung, die traditionell Teil der Subjektposition der Lehrenden sind, auch wenn sie sie überfordern. Die Integrität ihrer traditionellen Subjektposition ist aus 
dieser Perspektive also partiell in Frage gestellt und es sind diskursive Figuren der Automatisierung menschlicher Arbeitsleistung anschlussfähig, wie sie in vielen Kontexten im Hinblick auf Diskurse der «Digitalisierung» hervorgebracht werden. Gleichzeitig werden ihnen dadurch freie Kapazitäten in Aussicht gestellt, um diejenige Leistung zu erbringen, die bettermarks nicht erbringen kann. Der im Produktvideo skizzierte Horizont bleibt dabei jedoch beschränkt auf «andere Hilfestellungen», die weiterhin auf das Schliessen von «Wissenslücken» abzielen, statt z. B. auf gänzlich andere Ziele, andere Formen des Lernens oder zwischenmenschliche Arbeit. Hier wären alternative Konzepte der Subjektpositionierung - beispielsweise die Lehrperson als Care-Arbeiter/in, als menschliches Komplement des adaptiven Systems - denkbar, die aus dem System bettermarks selbst heraus nicht formulierbar scheinen. Eine weitere Veränderung der Subjektpositionierung der Lehrenden ergibt sich jedoch aus den Auswertungsmöglichkeiten, die bettermarks innen bietet.

\subsection{Auswertungen für Lehrpersonen}

In der Ansicht für Lehrpersonen finden sich mehrere Funktionen, die ihnen Auskunft über die Leistungsstände der Schülerinnen und Schüler geben. Das Dashboard liefert in erster Linie über die Ansichten «Überblick», «To Dos» und «Auswertung» inhaltliche Repräsentationen der Daten der Schülerinnen und Schüler. Unter «Überblick» sind verschiedene Statistiken zu finden, wer welche Aufgaben bearbeitet hat und mit welchem Erfolg.

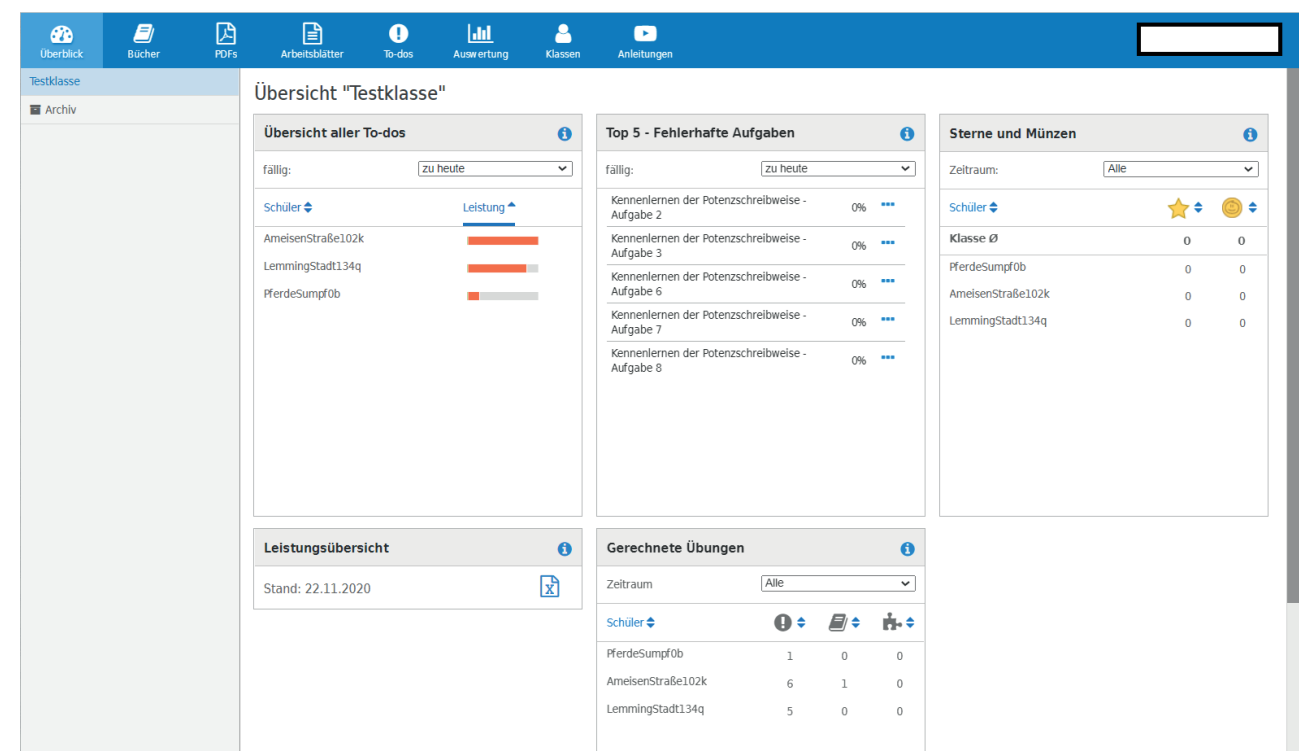

Abb. 9.: Screenshot des Überblicks-Dashboards für Lehrpersonen (Quelle: eigener Screenshot). 
Unter «To-dos» (Abb. 10) finden sich die an die jeweilige Klasse verteilten Aufgaben sowie eine Angabe, wie viele Schülerinnen und Schüler welche der Aufgabenbereiche bearbeitet haben sowie ein Kreisdiagramm, das anzeigt, wie viel des Bereichs sie bearbeitet haben und wie erfolgreich sie in Summe waren. Durch einen Klick auf die Zahl werden dann noch die Prozentzahlen der erfolgreich gelösten Aufgaben der individuellen Schülerinnen und Schüler angezeigt.

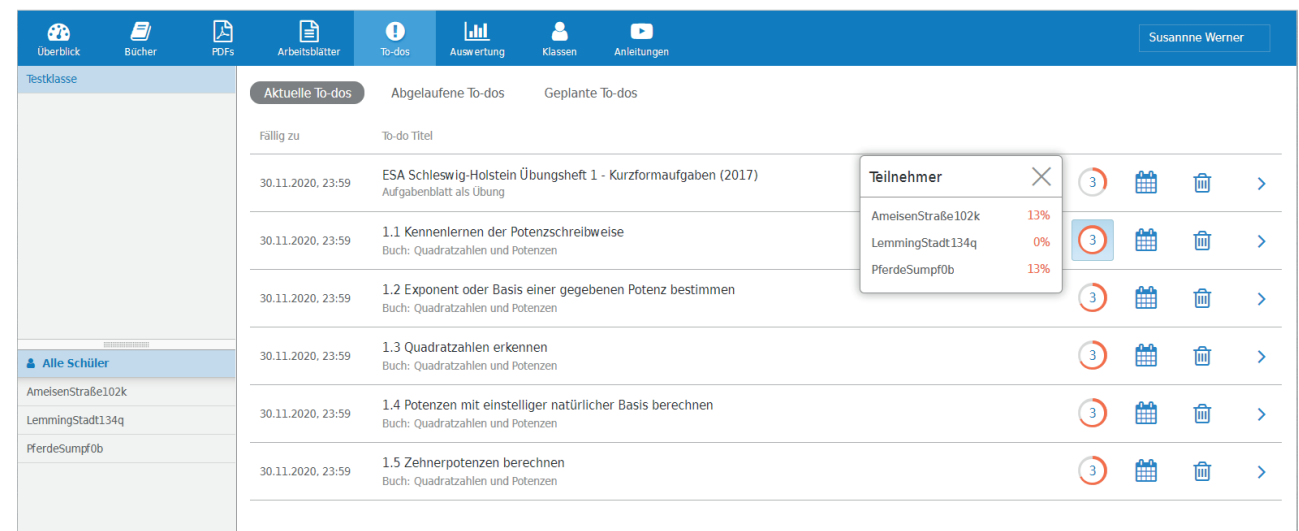

Abb. 10.: Screenshot der To-dos-Ansicht für Lehrpersonen (Quelle: eigener Screenshot).

Unter «Auswertung» können sich die Lehrpersonen insbesondere die vom System diagnostizierten «Wissenslücken» im Klassenschnitt sowie für einzelne Schülerinnen und Schüler anzeigen lassen.

«Und ich hab hier auch noch die Möglichkeit, die offenen Wissenslücken zu sehen, die es gerade in der Klasse gibt. Das heisst, hier kann ich auf die Wissenslücke klicken, sehen, welcher Schüler die hat, oder ich kann auch einen Schüler auswählen, und kann halt sehen, welche Wissenslücke noch nicht geschlossen worden ist. Bekomme hier auch ne Vorschau und wenn ich es beispielsweise schaffe, diese Wissenslücke mit dem Schüler in einer Einzelarbeit zu schliessen, und ich davon überzeugt bin, er muss das nicht mehr üben, dann habe ich auch die Möglichkeit, die Wissenslücke zu schliessen». (bettermarks 2018c, 2:48 - 3:18) 


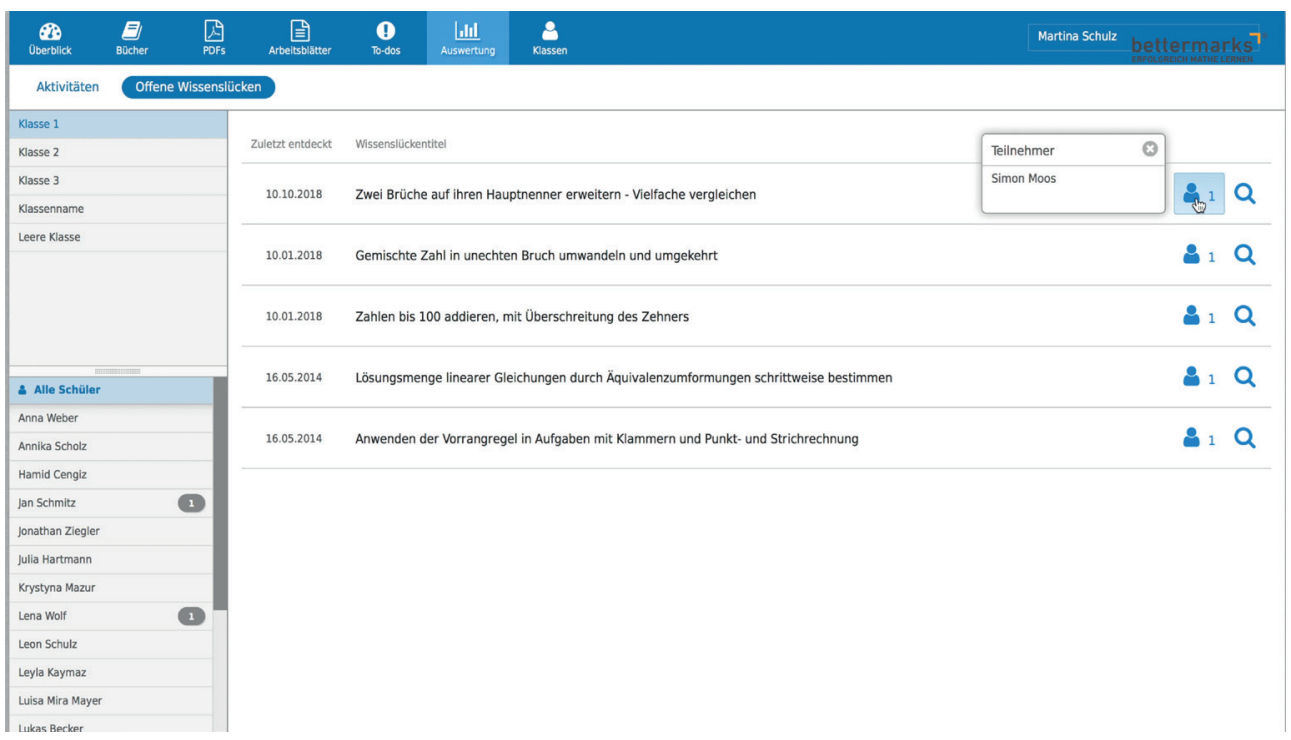

Abb. 11.: Auswertungs-Ansicht für Lehrpersonen (Quelle: bettermarks 2018c, 2:57).

Die Auswertungsfunktionen von bettermarks bringen Inhalte für die Lehrpersonen hervor, die in traditionellen Medienkonstellationen wenn nicht unmöglich, so doch unwahrscheinlich sind. So können Lehrpersonen zwar auch ohne bettermarks Aufgaben verteilen, die Ergebnisse einsammeln und sich statistische Auswertungen erstellen, doch wird das in den meisten Fällen vermutlich nicht getan zugunsten einer stichprobenartigen Überprüfung z. B. von Hausaufgaben. ${ }^{7}$ Das Dashboard generiert diese Inhalte ohne zusätzlichen Aufwand für Lehrpersonen, adressiert sie als Analystinnen und Analysten statistischer Daten und legt nahe, diese Daten als eine Grundlage für ihr didaktisches, methodisches und ggf. pädagogisches Handeln zu nutzen und dahingehend zu optimieren, die Statistiken zu verbessern. In das System eingeschrieben sind manageriale Wissensbestände und datenbasierte Managementpraktiken, die das Lehrpersonensubjekt als entsprechende Manager entwerfen. Am Beispiel einer «Vorwissen-Übung» erläutert Speroni derartige Erwartungen an die Nutzung der Daten:

«Sie als Lehrer können diese Vorwissen-Übung Ihren Schülern natürlich zuweisen, zum Beispiel, wenn Sie mit einem neuen Thema starten. Und gerade, wenn Sie eine neue Klasse haben, dann wissen Sie ja im Grunde gar nicht, wo die einzelnen Schüler stehen und was Sie nochmal wiederholen sollten. Und nach dieser Vorwissen-Übung wissen Sie ganz genau, wo die Defizite liegen und darauf können Sie reagieren.» (bettermarks 2018a, 3:29-3:49)

7 Hier ist darauf hinzuweisen, dass bereits das Stellen und die Abgabe von Hausaufgaben diese stichprobenartigen Kontroll-, Feedback- und Auswertungspraktiken teils hin zu systematischen verändert, wie aus weiteren Beobachtungen und Interviews in unserem Projekt deutlich wurde. 
Ähnlich wie schon für die Schülerinnen und Schüler, wenn auch weniger rigide, gilt auch hier, dass Lehrpersonen sich und ihre Lehrpraktiken optimieren, d. h. an das System und die von ihm generierten Inhalte anpassen, $d$. h. adaptieren sollen. Das Lernsystem kommt hier wieder als rational-objektive Instanz ins Spiel, die für die Tragfähigkeit der Diagnose bürgt und die Tatsache der oben bereits skizzierten Setzungen in den Hintergrund treten lässt.

Ebenfalls für die Subjektposition der Lehrperson relevant ist die Tatsache, dass sie die Möglichkeit haben, die vom System diagnostizierten «Wissenslücken» manuell zu «schliessen», was in diesem Kontext keine Veränderung der kognitiven Disposition einer Schülerin bzw. eines Schülers bezeichnet, sondern schlicht das Entfernen des Eintrags aus dem Profil der Schülerin bzw. des Schülers. Der Lehrperson wird also (noch) eine gewisse Urteilshoheit über den Lernstand der Schülerinnen und Schüler zugesprochen.

Das Verhältnis der Subjektpositionen von Lernenden und Lehrenden stellt sich dabei recht hierarchisch dar. Die Lehrpersonen haben permanent Einblick in die Eingaben der Schülerinnen und Schüler, die individuellen «Wissenslücken» und den Stand der Bearbeitung der Aufgaben. So wird ein Verhältnis der Kontrolle und Disziplinierung vorentworfen. Die Lernenden werden dabei als Profil sichtbar gemacht und als zu managendes Objekt in den Fokus gerückt, in dem die Schülerinnen- und Schüler-Subjekte nicht nur vom System, sondern vermittelt über das System (und seine spezifische Modellierung von Wissen, Lernen, Lernenden etc.) auch von der Lehrperson beurteilt werden.

Gleichzeitig haben Systeme wie bettermarks auch das Potenzial, die Lehrenden in die Subjektposition der Geprüften zu versetzen, insofern die datenförmige Auswertung der Leistung der Lernenden als Grundlage für die Qualität des Lehrangebots und damit der Lehrenden verwendet werden kann. Wenn z. B. Schulleitungen oder Eltern Einblick in die Auswertungen nehmen und bewerten, wie viele «Wissenslücken» vorhanden sind, wie viele geschlossen werden konnten etc., kommen neue aus- und bewertende Subjektpositionen hinzu und verändern die Subjektposition der Lehrenden innerhalb der Medienkonstellation dadurch grundlegend.

\section{Vertiefte kritische Überlegungen}

Abschliessend werden auf Grundlage der Analyse übergeordnete kritische Überlegungen zu bettermarks als Medienkonstellation angestellt. Ein zentraler Punkt, der sich durch nahezu alle Teile der Analyse von bettermarks gezogen hat, ist die (problem-)lösungsorientierte formale Modellierung der Praktiken des Lernens. Formalisierte Modellierung lässt sich zunächst auch auf lang etablierte Bestandteile im formalen Bildungssystem anwenden. Der Wissensstand der Schülerinnen und Schüler kann im System Schule beispielsweise durch Tests und Noten formal repräsentierbar 
gemacht werden, sogar in komputierbarer Form. Der entscheidende Unterschied bei Systemen wie bettermarks liegt im unbedingten Zwang zur Komputierbarkeit, der nicht-komputuierbare Elemente dessen, was Schule ausmacht - z. B. Beziehungsarbeit, Aushandlungen, Empathie, Umgang mit Diversität - systematisch ausschliesst. Dieser Zwang ergibt sich zum einen aus den materiellen Voraussetzungen der Computertechnologie, die bestimmte Formalisierungen erzwingen um Komputierbarkeit zu ermöglichen. Sie ergibt sich jedoch zum anderen auch durch Diskurse und Praktiken, die die Welt - und damit im Fall adaptiver Lernsysteme auch das Lernen - als ein über Komputation zu lösendes Problem modellieren. Wie Benjamin Jörissen schreibt, stehen derartige Praktiken «im Zeichen solutionistischer Effizienzoptimierung» und führen «neue[n] Logiken - nämlich technische Programmierlogiken» (Jörissen 2020, 343) ein. Solutionismus versteht er im Anschluss an Evgeny Morozov als das Bestreben, alle komplexen sozialen Zusammenhänge so umzudeuten, dass sie entweder als ganz genau umrissene Probleme mit ganz bestimmten, berechenbaren Lösungen oder als transparente, selbstevidente Prozesse erscheinen, die sich - mit den richtigen Algorithmen - leicht optimieren lassen» (Morozov 2013 zit. n. Jörissen 2020, 348). Dadurch ergibt sich Jörissen zufolge eine «Zuweisung von Positionen komputabler - also sichtbar gemachter - versus Positionen inkomputabler - also unsichtbar gemachter - Problemstellungen» und verbunden mit der hegemonialen Akzeptanz informatischer Modellierung eine «Vorentscheidung über Möglichkeit und Angemessenheit von Problemstellungen» (Jörissen 2020, 348). Das Lernen als Praktik, die Subjektpositionen der Lehrenden und Lernenden und auch die Lernziele und -inhalte werden damit zu den Bedingungen des tendenziell solutionistischen Computational Thinking entworfen (vgl. zu ähnlichen Überlegungen hinsichtlich Learning Analytics auch Selwyn 2019). ${ }^{8}$ Zwar wird sowohl im Kontext von Computational Thinking als auch informatischer Modellierung im Allgemeinen immer wieder betont, dass man sich der Tatsache bewusst ist, dass die nicht-komputierbaren Elemente ausgeschlossen werden und es dies zu reflektieren gilt (exempl. Wing 2006, 33; Modrow und Strecker 2016, 146f.). Doch verbleiben diese Ansätze im Horizont klassischer Modelltheorien, die davon ausgehen, dass Modelle als verkürzte Abbilder der Realität zu veranschlagen sind (exempl. Stachowiak 1973, 131f., explizit aufgegriffen z. B. in Modrow und Strecker 2016, 89), während aus medienwissenschaftlicher Sicht eher davon auszugehen ist, dass sie eigene Wirklichkeiten auf Grundlage bestimmter Diskurse konstituieren. Im Fall von bettermarks ist diese Wirklichkeit gekennzeichnet durch curricular vorgegebene und aufeinander aufbauende Lernziele, das «Durchschreiten» des «Lernzielnetzes» mittels IF/THEN/ELSE-Anweisungen, Modellierung der Schülerinnen- und Schüler-Subjekte als Kompetenzprofile, die Diagnose bzw.

8 Ben Williamson (2017) schreibt hinsichtlich der institutionellen Konsequenzen eines gesteigerten Einsatzes derartiger Systeme: «Educational data science and its applications are turning educational institutions (...) into metrological platforms that perform a constant scientific form of measurement of learning processes» (ebd., 121). 
Hervorbringung von «Wissenslücken» sowie Praktiken des eigenverantwortlichen «Schliessens» ebendieser im Sinne einer zielorientierten Optimierung. Hinzu kommen Kontroll- und Management-Praktiken auf Seiten der Lehrpersonen, die mit entsprechenden Subjektpositionierungen verbunden sind.

Im Hinblick auf Adaptivität ist bettermarks zwar ohnehin relativ beschränkt, da im Hintergrund das recht starre «Lernzielnetz» und vorgefertigte, regelbasierte Fehlerdiagnosen und Unterstützungsangebote liegen, steht aber in einem Punkt repräsentativ auch für komplexere Systeme: die Bindung an vorgegebene Lernziele. Durch sie werden insbesondere die Schülerinnen und Schüler zum eigentlichen «Gegenstand der Adaption» (s. o.; Lehmann 2010, 19), insofern die Adaption des Systems lediglich die Art und Weise betrifft, wie die Schülerin bzw. der Schüler dazu gebracht werden kann, sich so lange zu adaptieren, bis er oder sie das Lernziel erreicht hat (vgl. auch Weich 2018). Dadurch werden tendenziell behavioristische Lehr-Lernarrangements hervorgebracht, die bereits im Kontext kybernetischer Didaktik in den 1970er Jahren kritisch diskutiert wurden (exempl. Pongratz 1978). Dies ist zum Teil auf die skizzierten technisch-informatischen Voraussetzungen und Einschreibungen zurückzuführen. Gleichzeitig schliessen sie aber auch an lang tradierte schulische Prinzipien an, die sich ebenfalls mittels des skizzierten informatischen Abstraktionsund Formalisierungsprinzips modellieren lassen: die «task» besteht darin, Schülerinnen und Schülern durch die curricula bestimmtes Wissen zu vermitteln; und innerhalb des Schulsystems wird dies über formalisierte Repräsentationen wie Prüfungen, z. B. Klausuren, abgebildet. Der «output» spiegelt sich wiederum als Interpretation über das Erreichen der curricular gesetzten Wissensziele wider. Dieses technisch informatische Modellierungsprinzip und die damit einhergehende Optimierung der lernenden Individuen ist somit, unabhängig von neuen Softwarelösungen, bereits in das Bildungssystem Schule eingeschrieben. ${ }^{9}$

Diese Ähnlichkeiten zeigen: Während viele Mediendidaktikerinnen und Mediendidaktiker davon ausgehen, digitale Medien würden als «Trojaner» oder "Katalysator» per se zu einer «neuen Schule» mit progressiven (konstruktivistischen, konnektivistischen, ...) Konzepten von Lehren und Lernen führen oder sich gleichermassen für alte wie neue Konzepte als «Verstärker» eignen (Muuß-Mehrholz 2020), sind zumindest die in diesem Artikel skizzierten informatischen Wissensbestände und Praktiken - Computational Thinking, solutionistische Modellierung, IF/THEN/ELSE, Schleifen - strukturell anschlussfähig an behavioristische, kognitivistische Ansätze und lang tradierte schulische Prinzipien. Doch dieser Befund soll keinem Technikdeterminismus Vorschub leisten, sondern lediglich den technikfokussierten Fortschrittserzählungen der «Digitalisierungsdiskursen» (siehe kritisch hierzu u. a. Macgilchrist 2019

9 Eine diskursive und bildungspolitische Tendenz, die sich damit verknüpfen lässt, ist die von Biesta (2009) konstatierte «learnification», eine bildungsökonomische in dem Befund, dass im Rahmen der «Global Education Industry» bestimmte Prinzipien aus der IT-Industrie Einzug in Bildungsmedien und -institutionen halten (vgl. Verger et al. 2016). 
mit Bezug zu «Postdigitalität» als Gegenbegriff oder auch Dander 2020) auf eigenem Terrain begegnen. Ebenso sei erwähnt, dass schon seit vielen Jahren Vorschläge und technische Lösungsansätze formuliert werden, auch die curriculare Ebene der Lernziele z. B. über KI-Verfahren adaptiv zu gestalten, d. h. auch die Lernziele den Eigenheiten der Lernenden grundlegend anzupassen (exempl. Greer und Mandinach 1992, 87f.) und damit potenziell aus rückwärtsgewandten Lehr- und Lernkonzepten auszubrechen. Doch zum einen scheitern derartige Konzepte meist am Aufwand, der notwendig ist, um die Systeme aufzubauen und zum anderen an der Tatsache, dass das formale Bildungssystem eine derartige Adaptivität nicht vorsieht. Als Gedankenexperiment stelle man sich ein System im Schulkontext vor, das damit beginnt, zu versuchen, einer Schülerin oder einem Schüler Bruchrechnung beizubringen, durch die Adaptivität auf curricularer Ebene aber zu dem Schluss kommt, dass es für sie oder ihn passender wäre, statt Bruchrechnung Goethes Faust zu interpretieren oder gar alternative Schulformen zu erdenken oder politische Subversionsstrategien zu erproben. Sicher sind diese Beispiele polemisch und überspitzt, aber sie verdeutlichen doch die strukturelle Inkompatibilität zwischen bestimmten Adaptivitätsmöglichkeiten und dem formalen Bildungssystem.

\section{Ausblicke}

Um jedoch zumindest über einige tradierte Grenzen des Möglichen hinauszugehen, wären offenere und partizipativere Gestaltungsprozesse für adaptive Lernsysteme denkbar (anschliessen könnte man hier an Konzepte aus der partizipativen Gestaltung von Learning Analytics, exempl. Prieto-Alvarez, Martinez-Maldonado und Anderson 2019). Im Anschluss an Allert und Richter (2016) wären diese im Rahmen eines «transactional» Paradigma umsetzbar, während sich die Gestaltungsansätze von bettermarks und den meisten adaptiven Lernsystemen einem «instructional» Paradigma zuordnen lassen. Im transaktionalen Ansatz bedeutet Lernen

«not only [...] to acquire existing knowledge, which is prior to practice, but also to generate new knowledge and to productively cope with indeterminate situations. Creativity is closely related to uncertainty: Creative practice (creativity) is a mode of interaction in which individuals or collectives aim to cope productively with an indeterminate situation and bring forward new ideas.» (Allert und Richter 2016)

Auf der einen Seite ist eine solche Herangehensweise anschlussfähig an kreative Elemente der Problemlösung im Rahmen des Computational Thinking und artverwandter Ansätze wie dem Algorithmic Thinking. Doch auf der anderen Seite ist hier entscheidend, die solutionistische Fokussierung auf das Formalisier- und Komputierbare zu reflektieren und nicht bei der informatisch optimierten Modellierung 
der Welt stehen zu bleiben, sondern darüberhinausgehende Elemente mitzudenken und zu gestalten - auch (z. B. curriculare) Elemente des Schulsystems und der schulischen Medienkulturen. Lernsysteme, die einem transaktionalen Paradigma Rechnung tragen, müssten durch ihre Modellierung Adaptivität und die Prozessierung von Unbestimmtheiten ermöglichen und in der Lage sein, die vielfältigen Praktiken und Ziele der Lernenden möglichst optimal in der Sphäre des Komputierbaren zu repräsentieren und gleichzeitig in Medienkonstellationen eingebettet werden, die Räume für die Gestaltung des Nichtformalisierbaren und -komputierbaren eröffnen. ${ }^{10}$

Die kritische Reflexion müsste sich dabei nicht nur auf algorithmische Optimierungskonzepte innerhalb der Repräsentationen der informatischen Modelle beziehen, wie sie exemplarisch anhand von bettermarks herausgearbeitet wurden. Sie müsste auch die Optimierung der Repräsentation selbst kritisch in den Blick nehmen, d. h. nicht nur auf eine möglichst optimale Repräsentation von Zielen und Praktiken oder möglichst umfassende Adaptivität schauen, sondern auch die Grenzen der komputierbaren Repräsentation an sich. Ein solches Vorhaben wäre zweifellos sehr ambitioniert und angesichts sowohl des oben skizzierten technisch-konzeptionellen Aufwands als auch der institutionellen Rahmenbedingungen nur in bestimmten Grenzen umsetzbar. Notwendig wären dafür partizipative Forschungs- und Gestaltungsprozesse, die sowohl Lehrende und Lernende als auch Wissenschaftlerinnen und Wissenschaftler aus Bildungsmedien- und Lernforschung sowie Informatikerinnen und Informatiker auf Augenhöhe zusammenarbeiten liesse (vgl. für einen ähnlichen Vorschlag hinsichtlich Learning Analytics im Hochschulbereich Selwyn 2020).

Vorschläge, Endnutzer und Endnutzerinnen stärker in den Softwareentwicklungsprozess zu integrieren, existieren schon seit mehreren Jahrzehnten (vgl. Raum und Baronick 1991). Dieses Bestreben findet sich auch in dem Bereich der agilen Softwareentwicklungsprozesse wieder. So definieren Beck et al. in ihrem «Manifesto for agile software development» einen ihrer vier Kernwerte als «Customer collaboration over contract negotiation» (Beck et al. 2001). Während Cockburn für seine agile Softwareentwicklungsmethode Crystal Clear explizit die Rolle der Nutzenden als Teil des Entwicklungsteam angibt und zusätzlich als eine von sieben Richtlinien «There is direct user involvement» anführt (Cockburn 2002). Agile Softwareentwicklungsmethoden haben heute zwar verbreitet Einzug in die Industrie gefunden, so geben Honda et al. an «agile is now the mainstream software development method of choice worldwide» (Honda et al. 2018). Nichtsdestotrotz geben aber in einer Umfrage $84 \%$ der Organisationen an, dass ihre agilen Arbeitsprozesse noch reifen (Honda et al. 2018).

10 Damit zusammenhängende wichtige Aspekte sind die Situierung und Kontextualisierung der lernbezogenen Daten sowie die Auseinandersetzung mit ihnen (vgl. Bachmair et al. 2018). 
An diese Traditionen und Tendenzen kann zunächst angeschlossen werden. Dabei ist jedoch zum einen zu reflektieren, dass sie die oben skizzierte solutionistische Haltung fortsetzen und in gewisser Weise die Zufriedenheit der Nutzenden mit dem Endprodukt als zu lösendes Problem veranschlagen. Zum anderen ist zu reflektieren, welche Aspekte in einem derartigen Entwicklungsprozess zum Thema gemacht werden. Die partizipative Reflexion der Modellierungen der Bedürfnisse und Realitäten der Nutzenden müsste nicht nur didaktische und methodische Aspekte beinhalten, sondern auch schul- und medienkulturelle, die die diskursiven Einschreibungen, Möglichkeitsbedingungen für Inhalte, Subjektpositionierungen und (auch implizite) Praktiken zunächst an die Oberfläche bringen und dann nicht nur funktionalistisch, sondern auch kritisch diskutieren. In unserer Nachwuchsforschungsgruppe «Postdigitale Medienkonstellationen in der Bildung» am Georg-Eckert-Institut möchten wir daher agile Prozesse aufgreifen, aber mit kritischen medienwissenschaftlichen Analysen verbinden. Hierbei müssen nicht nur die im vorliegenden Artikel eingenommenen Perspektiven berücksichtigt, sondern umfassende Medienkonstellationsanalysen zugrunde gelegt werden, die die Praktiken und Situierungen der Beteiligten in Betracht ziehen und auch die Ebene der technischen Implementierung nicht nur an der Oberfläche, sondern tiefgehender einbeziehen. Daher erforschen und erproben wir derartige Prozesse. Die zu Beginn des Textes aus unserer Forschung aufgegriffenen Aussagen von Lehrpersonen sowie Schülerinnen und Schülern zu Optimierungspotenzialen und Kritik hinsichtlich adaptiver Lernsysteme könnten vor diesem Hintergrund mit den im Rahmen des Artikels herausgearbeiteten medienkulturwissenschaftlichen Analysen und informatischen Gestaltungsmöglichkeiten kombiniert werden. Aus den jeweiligen Zwängen und Logiken könnte ein solcher Gestaltungsprozess sicher nur bedingt herausführen, doch würde er sie zumindest transparent und diskutierbar machen, um fundierte und ausgehandelte Design-Entscheidungen treffen zu können.

\section{Literatur}

Allert, Heidrun, Michael Asmussen, und Christoph Richter. 2018. «Formen von Subjektivierung und Unbestimmtheit im Umgang mit datengetriebenen Lerntechnologien - eine praxistheoretische Position». Zeitschrift für Erziehungswissenschaft 21: 142-158. https://doi. org/10.1007/s11618-017-0778-7.

Allert, Heidrun, und Christoph Richter. 2016. «A Framework for Learning Analytics: Paradigms and Practices». Researchgate. https://doi.org/10.13140/RG.2.2.33107.17446.

Bachmair, Benjamin, Keith Turvey, John Cook, und Norbert Pachler. 2018. «Learning analytics and its metrics - approaching an educational frame via a social semiotic pathway». medienimpulse 56 (3). https://doi.org/10.21243/mi-03-18-10. 
Ball, Stephen J., und Antonio Olmedo. 2013. Care of the self, resistance and subjectivity under neoliberal governmentalities. Critical Studies in Education 54(1), 85-96. https://doi.org/10 .1080/17508487.2013.740678.

Beck, Kent, Mike Beedle, Arie Van Bennekum, Alistair Cockburn, Ward Cunningham, Martin Fowler, James Grenning, Jim Highsmith, Andrew Hunt, Ron Jeffries, Jon Kern, Brian Marick, Robert C. Martin, Steve Mellor, Ken Schwaber, Jeff Sutherland, und Dave Thomas. 2001. «Manifesto for agile software development». https://agilemanifesto.org.

Bettermarks. 2018a. «Wissenslücken». https://www.youtube.com/watch?v=lvA5wXyaY9c.

Bettermarks. 2018b. «So lernen Schüler mit bettermarks». https://www.youtube.com/ watch?v=hOC $1 \mathrm{mrmDMYQ}$.

Bettermarks. 2018c. «Auswerten der Ergebnisse». https://www.youtube.com/ watch?v=0YBesKKCdkc.

bettermarks. 2020a. Über uns. https://de.bettermarks.com/ueber-uns/.

Biesta, Gert. 2009. «Good Education in an Age of Measurement: On the Need to Reconnect with the Question of Purpose in Education». Educational Assessment Evaluation and Accountability 21(1). https://doi.org/10.1007/s11092-008-9064-9.

Bradbury, Alice, und Guy Roberts-Holmes. 2017. The Datafication of Primary and Early Years Education: Playing with Numbers. Abingdon, Oxon; New York, NY : Routledge, Taylor \& Francis Group.

Bulger, Monica. 2016. «Personalized Learning: The Conversations We're Not Having». Data \& Society Working Paper. https://datasociety.net/pubs/ecl/PersonalizedLearning_primer_2016.pdf.

Chen, Peter Pin-Shan. 1976. "The Entity-Relationship Model. Toward a Unified View of Data». ACM Transactions on Database Systems, Vol. 1, No. 1: 9-36. https://doi. org/10.1145/320434.320440.

Cockburn, Alistair. 2002. Agile Software Development. Boston: Addison-Wesley.

Dander, Valentin. 2020. «Sechs Thesen zu Verhältnis von Bildung, Digitalisierung und Digitalisierung». In Digitalisierung - Subjekt - Bildung. Kritische Betrachtungen der digitalen Transformation, herausgegeben von Valentin Dander, Patrick Bettinger, Estella Ferraro, Christian Leineweber und Klaus Rummler, 19-37. Berlin \& Toronto: Verlag Barbara Burdrich. https:// doi.org/10.2307/j.ctvvb7n3h.5.

Döbeli Honegger, Beat. 2017. Mehr als 0 und 1. Schule in einer digitalisierten Welt. 2. Auflage. Bern: hep Verlag.

Eickelmann, Birgit, Wilfried Bos, Julia Gerick, Frank Goldhammer, Heike Schaumburg, Knut Schwippert, Martin Senkbeil, und Jan Vahrenhold, Hrsg. 2019. ICILS 2018 \#Deutschland Computer- und informationsbezogene Kompetenzen von Schülerinnen und Schülern im zweiten internationalen Vergleich und Kompetenzen im Bereich Computational Thinking. Münster: Waxmann. https://doi.org/10.25656/01:18166. 
Fenwick, Tara, Eric Mangez, und Jenny Ozga. 2014. Governing knowledge: Comparison, knowledgebased technologies and expertise in the regulation of education. London: Routledge.

Gießmann, Sebastian. 2014. Die Verbundenheit der Dinge. Eine Kulturgeschichte der Netze und Netzwerke. Berlin: Kulturverlag Kadmos.

Greer, Jim und Ellen Mandinach. 1992. «Representing Curriculum and Designing Instructional Tasks». In Adaptive Learning Environments. Foundations and Frontiers, herausgegeben Herausgegeben von Marlene Jones und Philipp H. Winne, 87-89. Berlin/Heidelberg/New York/ London/Paris/Tokyo/Hong Kong/Barcelona/Budapest: Springer.

Groff, Jenny. 2017. Personalized learning: The state of the field \& future directions. Center for Curriculum Redesign.

Hansen, Marit, Meiko Jensen, und Martin Rost. 2015. «Protection Goals for Privacy Engineering». 2015 IEEE Security and Privacy Workshops: 159-166. https://doi.org/10.1109/ SPW.2015.13.

Hoda, Rashina, Norsaremah Salleh, und John Grundy. 2018. «The Rise and Evolution of Agile Software Development». IEEE Software PP. 1-1. https://doi.org/10.1109/MS.2018.290111318.

Jörissen, Benjamin, und Winfried Marotzki. 2009. Medienbildung - Eine Einführung. Bad Heilbrunn: Klinkhardt (UTB).

Jörissen, Benjamin. 2020. «Ästhetische Bildung im Regime des Komputablen». Zeitschrift für Pädagogik 66 (3): 341-356.

Krommer, Axel. 2013. «Von Skinners „Teaching Machines“ (1954) zu den „Learning-Apps“». https://axelkrommer.com/2013/09/17/von-skinners-teaching-machines-1954-zu-den-learning-apps/.

Lehmann, Robert. 2010. Lernstile als Grundlage adaptiver Lernsysteme in der Softwareschulung. Münster / New York / München / Berlin: Waxmann.

Macgilchrist, Felicitas. 2019. «Digitale Bildungsmedien im Diskurs. Wertesysteme, Wirkkraft und alternative Konzepte». Aus Politik und Zeitgeschichte: Bildung und Digitalisierung APuZ. 18-23. https://www.bpb.de/apuz/293124/digitale-bildungsmedien-im-diskurs.

Modrow, Eckart, und Kerstin Strecker. 2016. Didaktik der Informatik. Oldenbourg: De Gruyter.

Morozov, Evgeny. 2013. Smarte neue Welt: Digitale Technik und die Freiheit des Menschen. München: Blessing.

Muuß-Mehrholz, Jöran. 2020. «Trojaner, Katalysator oder Verstärker?» Lernende Schule Nr. 91 / 2020, Unterricht digital.

Paramythis, Alexandro, und Susanne Loidl-Reisinger. 2004. «Adaptive Learning Environments and eLearning Standards». Electronic Journal of E-Learning 2 (1): 181-194. https://academic-publishing.org/index.php/ejel/article/view/1458.

Pongratz, Ludwig A. 1978. Zur Kritik kybernetischer Methodologie in der Pädagogik. Ein paradigmatisches Kapitel szientistischer Verkürzung pädagogisch-anthropologischer Reflexion. Frankfurt am Main/Bern/Las Vegas: Peter Lang. 
Poole, David, und Alan Mackworth. 2017. Artificial Intelligence: Foundations of Computational Agents, second edition, Cambridge: Cambridge University Press.

Priedigkeit, Marvin, Andreas Weich, und Ina Schiering. 2021. «Learning Analytics and Privacy - Respecting Privacy in Digital Learning Scenarios». Privacy and Identity Management. Privacy and Identity 2020. IFIP Advances in Information and Communication Technology 619: 134-50. https://doi.org/10.1007/978-3-030-72465-8_8.

Prieto-Alvarez, Carlos G., Roberto Martinez-Maldonado und Theresa Dirndorfer Anderson. 2019. «Co-Designing Learning Analytics Tools with Learners». In Learning Analytics in the Classroom. Translating Learning Analytics Research for Teachers, herausgegeben von Jason M. Lodge, Jared Cooney Horvath und Linda Corrin, 93-110. London und New York: Routlege.

Raum, Harald, und Sabine, Baronick. 1991. Partizipative Softwareentwicklung. In SoftwareErgonomie '91. Berichte des German Chapter of the ACM, herausgegeben von D. Ackermann und E. Ulich. Wiesbaden: Vieweg+Teubner Verlag. https://doi.org/10.1007/978-3-32294654-6_12.

Reinmann Gabi. 2013. «Didaktisches Handeln. Die Beziehung zwischen Lerntheorien und Didaktischem Design». In L3T. Lehrbuch für Lernen und Lehren mit Technologien. 2. Auflage, herausgegeben von Martin Ebner und Sandra Schön. Berlin: E-Publi [12] S. https://nbnresolving.org/ urn:nbn:de:0111-opus-83381.

Selwyn, Niel. 2019. «What's the Problem with Learning Analytics?». Journal of Learning Analytics 6 (3): 11-19. https://doi.org/10.18608/jla.2019.63.3.

Selwyn, Neil. 2020. «Re-imagining 'Learning Analytics' ... a case for starting again?». The Internet and Higher Education 46. https://doi.org/10.1016/j.iheduc.2020.100745.

Stachowiak, Herbert. 1973. Allgemeine Modelltheorie. Wien: Springer-Verlag.

Schröter, Jens. 2004. Das Netz und die Virtuelle Realität. Zur Selbstprogrammierung der Gesellschaft durch die universelle Maschine. Bielefeld: transcript. https://doi. org/10.14361/9783839401767.

Troeger, Jasmin (in Vorbereitung). Der datafizierte Unterricht. Eine multimodale diskursanalytische Betrachtung präfigurierender Datenpraktiken adaptiver Lernsoftware. Unveröffentlichte Dissertation, Universität Leipzig, Leipzig.

Verger, Antoni, Gita Steiner-Khamsi, und Christopher Lubianski. 2016. «The Emergence and Structuring of the Global Education Industry: Towards an Analytical Framework». In World Yearbook of Education 2016: The Global Education Industry, herausgegeben von Antoni Verger, Gita Steiner-Khamsi, und Christopher Lubianski. London und New York: Routledge.

Weich, Andreas. 2017. Selbstverdatungsmaschinen. Zur Genealogie und Medialität des Profilierungsdispositivs. Bielefeld: transcript. https://doi.org/10.14361/9783839439425.

Weich, Andreas. 2018. «Was nicht passt, wird passend gemacht. Learning Analytics als Teil des Profilierungsdispositivs». Medienimpulse, 1/18. https://doi.org/10.21243/mi-01-18-04. 
Weich, Andreas. 2020. «Digitale Medien und Methoden: Andreas Weich über die Medienkonstellationsanalyse». Open-Media-Studies-Blog, 16.06.2020. https://www.zfmedienwissenschaft.de/online/open-media-studies-blog/digitale-medien-und-methoden-weich.

Weich, Andreas, Katja Koch, und Julius Othmer. 2020. «Medienreflexion als Teil „digitaler Kompetenzen» von Lehrkräften? Eine interdisziplinäre Analyse des TPACK- und DigCompEduModells». k:ON - Kölner Online Journal für Lehrer*innenbildung 1, 1/2020: 43-64. https://doi. org/10.18716/ojs/kON/2020.1.3.

Williamson, Ben. 2015. «Digital education governance: Data visualization, predictive analytics, and 'real- time' policy instruments». Journal of Education Policy 31(2), 123-141. https://doi. org/10.1080/02680939.2015.1035758.

Williamson, Ben. 2017. Big Data in Education. The digital future of learning, policy and practice. Los Angeles et al: Sage.

Wing, Jeanette M. 2006. "Computational Thinking». Communications of the ACM, March 2006/ 49 (3): 33-35. https://doi.org/10.1145/1118178.1118215.

Winkler, Hartmut. 1997. Docuverse. Zur Medientheorie der Computer. München: Boer. 